\title{
Research on Content Integration of University Journal in Dissemination*
}

\author{
Meiying Wu \\ City College of Dongguan University of Technology \\ Dongguan, China 523419
}

\begin{abstract}
University journals are an important part of academic journals. To improve the efficiency of digital dissemination of university journals and meet the reading needs of users in the information age, it is necessary to solve the problem of content integration in the dissemination of university journal. Based on analysis of the current situation and existing problems of the content integration of China university journals, this paper proposes three suggestions on the content integration of university journal: setting a clear goal of content integration; having the whole-course awareness of integration to make content integration throughout the production of contents; attaching importance to the improvement of editorial quality to facilitate the successful completion of content integration.
\end{abstract}

Keywords-university journal; dissemination; content integration

\section{INTRODUCTION}

Sprouting in the late 19th and early 20th century, university journals, through over 100 years of development, now grown from a handful to more than 2,500 categories today, accounting for $1 / 4$ of the total periodicals of the county, and has become an important part of academic journals in China. In the initial period of founding, especially in the 1980s, university journals made great contributions to the development of higher education and to the prosperity of academic cause. With the deepening of social reform, the continuous development of information technology and the increasingly rising of users' requirements for the dissemination speed of academic information, the digital dissemination of university journals are getting more important. However, throughout the university journals in recent years, although we have made some achievements in accelerating the dissemination speed of academic information and realized digitization to a certain degree, there are still some problems there [1-2]. In terms of solving problems of university journal dissemination, people have reached a consensus about using advanced information technology and selecting multi-channel transmission way, but little attentions has been paid to content integration in the process of dissemination. Therefore, the author intends to make useful studies on the content integration

*Fund project: 2016 Editorial Research Project of University Journal Research Association of Guangdong (No.:20160203); 2016 Youth Teacher Development Fund Project of City College of Dongguan University of Technology, Dongguan, Guangdong (No.: 2016QJY016R); 2017 Key Platform and Scientific Research Project of Guangdong (No.: 2017WQNCX185). of college journals

\section{INTEGRATION OF DISSEMINATION CONTENT AND TYPE OF UNIVERSITY JOURNAL}

\section{A. Concept of Content Integration}

Influenced by developing trend of media integration, the content presentation form based on pure text and diagram cannot meet the reading needs of readers, which requires the disseminators of university journals conduct further processing of the communication contents, that is, content integration. Content integration is different from content production. Content production of the university journal refers to the process that the editor, after topic selection, contribution soliciting, contribution screening, editting and processing, turns the original content into high-quality content and publishes it in the university journal. The author holds that in university journals, content integration refers to the process of merging, mining, sorting and reorganization of various academic content data in different forms of presentation so as to form new content information. After content integration, the content products of university journal can realize the second use, which, to a certain extent, increases the amount and coverage of transmission information, and moreover, the content after reorganization can better satisfy the requirements of various transmission ways, so as to improve the effect of dissemination of university journals. Therefore, content integration is an important guarantee for university journals to improve communication efficiency.

\section{B. Types of Content Integration}

In terms of content integration, some scholars, from the perspective of the use of mobile magazine platforms, focusing on four aspects including content quality, utilization ways, presentation method and service experience, classify content integration into three types-- content roughing, content detailing and integration, content value addition and exploration [3]. This method of classification places emphasis on the types of content integration. To a disseminator of university journal, its primary concern is how to realize content integration. According to the practice situation of content integration of academic journals at home and abroad in the dissemination, the author classifies content integration into two types: digitalized integration and diversified integration. 
1) Digitalized integration: Digitalized integration is to realize the digitalization of the content of university journals so as to realize the dissemination of academic contents via the digital dissemination means and ways. The digitalized integration way includes two stages: initial stage and further digitalization stage.

First, it is the initial stage. The disseminator only needs to conduct simple format transformation and classification of the printed academic content. In this stage, the reader can click the classified literature by steps to view the information it wants, for example, according to the year, period, piece, etc.; or directly input the keywords such as the author's name, article name, theme, etc. to get the targeted literature. The advantage of this is that it breaks the fetters of paper carrier, so that readers can read if the reading terminal device with internet access is available. Moreover, the data capacity is large, so that readers can not only read the latest journal content but also can read other digitalized content, but the content dissemination of this stage is characterized by passivity. That is, if the readers do not search, the content they are interested in will not show up automatically. To solve the problem and offer readers more intellectuality services, it is required to conduct further processing of these data.

Second, it is the further digitalization stage. Based on the first stage, this stage establishes an organic connection between the disseminator and the content, the content and the content, the content and the reader, the disseminator and the reader by using computer programming and then actively offers services to readers via these connections. For example, the disseminator, according to the reader's the degree of mastery of the academic content, recommends academic papers of high creativity, high readability and high quality to the reader; when the reader is reading a piece of paper, the disseminator can actively push other related papers to the reader, etc. Through further digitalized content integration, the disseminator can offer readers more intellectuality services to bring readers more convenient reading experience. In the meantime, to some extent, this can alleviate the problem that the professional academic content provided by the university journal of each issue is too little to meet readers reading needs.

In the further digitalized stage, although this kind of digitalized content integration offers convenience for readers, the content is the same as that in the printed university journal, so the single content presentation mode cannot meet the requirements of readers for diversified content. To solve this problem, it is required to make further creative integration.

2) Diversified integration: The diversified integration is to conduct diversified processing and utilization of the digitalized content, which requires the disseminator to make further sorting, mining and reorganization of the dissemination content so as to provide more content valueadded services, such as shortening the long academic papers into short ones, introducing the latest research achievements in the form of news report, and adding abundant multimedia materials. The creative integration way has been widely applied in some well-known academic journal websites abroad. Taking the journal Science website for example, in the column Editors' Choice, the editor recommends academic contents to readers in a way of introducing the main information of the article by using its own expression instead of directly copying and pasting the original article so as to highlight the innovation point of the article; in the column Sciencelnsider, the editor introduces the readers to the latest scientific information in the research field in the form of news report. The advantage of diversified integration is to realize the diversification and flexibility of the content dissemination ways to effective remedy the defect that readers are easy to feel bored when reading traditional academic papers, and in the same time, the diversified integration also meet readers' demand for fragmentization reading, to some extent. However, its disadvantage is that it is difficult to integrate some contents, which raises higher requirements for the resource allocation of the editorial office and for the editor' quality.

\section{ANALYSIS OF THE EXISTING PROBLEMS OF CONTENT INTEGRATION}

\section{A. Simpleness, Repetition and Lack of Differentiation of the Dissemination Content}

At present, the contents of university journals are disseminated via paper medium, various database platforms, PC internet website and mobile phone terminal. Each way of dissemination has its own characteristics and has different requirements for the content integration. For example, the traditional paper medium dissemination, although with long transmission cycle, is essential. The paper university journal disseminates not only the academic content, but also the academic image of the whole journal, so it realizes the integrated dissemination of the brand of the academic journal. The content production of paper university journals is the basic link of content integration. The content dissemination via various database platforms is a common way of the dissemination of university journals, currently, and its dissemination content is the same as the paper dissemination content. The content dissemination via mobile phone terminal is mainly to cater the needs of readers for mobile reading and fragmented reading, so the content should not be too long, and this requires differentiation adjustment of the dissemination content. The content dissemination via PC internet website integrates the advantages of many dissemination ways: first, rapid speed of dissemination; second, no limitation of paper layout capacity; third, diversification of content forms. However, at present, for most university journals, no matter which dissemination way is adopted, the contents of dissemination are basically the same, and no proper integration of content is made to adapt to different dissemination ways. For example, the content dissemination via PC internet website, the journals and even the websites of most universities adopt the same construction template, whose functions are nothing more than current directory lookup and back issue lookup, and contents of dissemination are the same with those of the paper journals. 


\section{B. Single Content Presentation Form and Reading Experience}

The presentation forms of content are varied. The same content can be recorded via text description and also can be presented via diagrams, and with the development of information technology, it can also be recorded via voice, animation, video, etc. For university journals, they have advantaged conditions to realize diversified content presentation forms, and PC internet website is a good communication platform. However, most university journal websites fail to achieve this at present, as words, pictures and forms are still the only way for them to present the content, which can only provides readers with single reading experience.

\section{Few Value-added Services of Content}

The core content of value-added services is according to customers' needs to provide them with services beyond the scope of normal services, or to offer services by adopting unusual service methods [4]. The term content value-added services here refers to additional services in addition to the content itself, including the selection publishing function in the publication of some university journals; the keyword search function based on establishment of a database; multilevel menu service function provided in WeChat service official account, as well as recommending excellent articles, providing searching and reading ranking, etc. The more value-added services provided, the more surprises brought to readers and the more conducive it is to the content dissemination of university journal. At present, however, few university journals provide these value-added services. Therefore, it is worth considering how to provide more value-added services for readers in the content integration of university journals.

\section{STRATEGY FOR PROMOTING CONTENT INTEGRATION IN THE DISSEMINATION OF UNIVERSITY JOURNALS}

From the current situation of content integration of university journal in dissemination, there is still a long way to go for university journals in content integration. To promote content integration, the following measures shall be taken.

\section{A. Setting a Clear Goal of Content Integration}

With the advent of the era of big data, information updating is speeding up, so if the information cannot attract readers' attention quickly, it will be submerged by the information ocean in an instant. Therefore, the operation of university journal has to paying equal attention to both the content and the reader from the original "content is king" [5].For the university journals, their contents of dissemination also have to meet the psychological needs of readers. For this purpose, the content integration of university journals shall reach the following five goals: 1 . The content shall be professional. University journals as the periodicals for showing the academic achievements, their contents mainly are original research articles, reviews, etc. and their readers are mostly researchers who read these articles to obtain professional information in a research field. Thus, for university journals, the information content of dissemination shall reflect the expertise. 2. The content shall be different from and complementary with that of the printed university journal. For example, readers follow the WeChat account of the journal for the purpose of obtaining more professional academic information that is different from and complementary with that of the printed university journal, but if the information they can obtain are the same, it will lose practical significance from the perspective of information dissemination. 3. The content presentation forms shall be diversified. At present, media integration has imposed a great influence on readers' reading habits, which is mainly reflected in the diversity of readers' demands for content presentation forms. The former planar texts and diagrams cannot meet the needs of the readers, which requires the university journals to realize diversified content presentation methods in the information dissemination. 4. Realizing the interactivity in the process of dissemination. In the process of reading, readers have the needs of information feedback, in addition to obtaining information. Therefore, in the process of information content dissemination, the university journal is required to provide readers with a information feedback channel which shall be followed up and maintained by professional person to avoid it becoming a mere formality. 5. Provide all-dimensional value-added services for readers. Understanding readers' reading habits and needs is the key to providing value-added services. For example, apart from releasing some academic research information, it also can add other functions such as online periodicals and articles searching, online office, selection publishing, etc. to provide convenience for readers to realize mobile reading, for authors to inquire information of their contributions, and for editors to realize mobile office, etc.

\section{B. Having the Whole-course Awareness of Integration to Make Content Integration Throughout the Production of Contents}

When you set a clear goal content integration, the following problem to be solved is about how to reach the goal. To achieve high-level content integration, it is impossible to accomplish in an action, so we cannot only complete the work in a certain time but have to have the whole-course awareness of integration. The whole-course awareness of integration is to make the integration awareness permeate every link of content production, collection and presentation [6]. This requires the editors of university editors to conduct the content integration throughout the whole course of "topic selection-contributions soliciting - article reviewing - editing- publishing". For example, in the work of topic selection and contributions soliciting, university journal editors are required to understand the current academic trends in a certain field, find relevant materials, and then start to organize excellent articles. In the past, this work was mainly done offline, but now, to strengthen the depth of content integration and obtain more dissemination content, editors can try to complete this work both offline and online at the same time. They can select relevant materials and put them on the website or WeChat public platform, and then organize a topic discussion to expand the scope of influence of the topic strategy. In this way, it can not only attract excellent contributions, but also can activate the contents in the website 
or WeChat public platform to make them different from the paper version. For another example, in the handling of contributions, the editors were mainly focusing on the paper publication, so when receiving excellent contributions, they mainly collect written materials, but now, considering the requirements for the diversified content presentation forms in the goal of content integration, the editors may strengthen the connection with the authors and ask them to provide other more materials apart from the contribution as much as possible, and then upload them according to different dissemination channels. This not only can make the dissemination content more diversified and but also can better meet readers' demand for diversified reading. Moreover, after the publication of the contribution, editors in the past only need to upload the article to websites or publishing platforms, but now to provide more value-added services, they are required to strengthen the management of the manuscripts on the basis of original work, so as to provide convenience for readers to search.

\section{Attaching Importance to the Improvement of Editorial Quality to Facilitate the Successful Completion of Content Integration}

In the dissemination of university journals, editors, as engineers of content production, are playing an important role in the process of content integration. With society development, new media are increasingly applied in the publication of university journals, so the editors of university journals are facing more complicated problems in the process of publication then before. Therefore, editors are required to improve their quality accordingly. Apart from the abilities of professional research, editing affairs and language and words processing, editors should also have the following qualities: (1) the ability of message editing and releasing via PC or mobile terminal. There is a big difference between information releasing via $\mathrm{PC}$ or mobile terminal and traditional information releasing via paper medium, and apart from different releasing means, the both have different requirements for contents. Thus, first of all, editors need to understand the principles of information publishing via PC or mobile terminal, and have a good command of the corresponding information publishing skills. Secondly, they need to learn content editing skills so that the edited information can better meet the psychological needs of readers' for mobile reading, such as short and concise information, with appropriate illustrating pictures, videos, etc. (2) The ability of information acquisition and accurate information extraction. In the era of big data, editors can no longer wait for information content to come itself, but they need to take the initiative to acquire information. First, editors have to master the ways and methods of information acquisition and use modern internet tools to search for information. Second, editors should develop sharp observation and discrimination so as to extract useful information from the massive amounts of information contents. 3. Editors should have higher interpersonal communication ability and higher awareness of social cooperation. In view of the reconstruction of editors' quality, the university journal office or editorial office are supposed to pay more attention to the training of editors' quality and offer more opportunities to the editors to go out to know more about the new environment and receives more new knowledge, so as to broaden their view and improve their ability.

\section{CONCLUSION}

At present, the dissemination of university journals has been paid more and more attention by the academic circle. In terms of solving problems of university journal dissemination, people have reached a consensus about selecting multi-channel transmission way and accelerating media integration, but little attentions has been paid to content integration in the process of dissemination. Therefore, the author raises the problem in this paper to start a discussion and seek advice from people of insight.

\section{REFERENCES}

[1] Zhang Haisheng, Cai Zongmo, Wu Zhaoping. Media Integration and Development of Academic Journals: Process, Problem and Prospect [J] Chinese Editors, 2018(01):76-82. 张海生,蔡宗模,吴朝平.学术期刊媒 体融合发展: 历程、问题与展望[J].中国编辑,2018(01):76-82

[2] Liu Jin, Tian Yu, Li Lanxin. Media Integration Dilemma of Academic Journals and Solution [J]. Acta Editologica, 2018(01):4-7. 刘津,田雨,李 兰欣. 学术期刊媒体融合发展困局与破局之策 $[\mathrm{J}]$. 编辑学 报,2018(01):4-7.

[3] Meng Xiangzhao. Research on Content Integration Mechanism and Optimization Strategy of Mobile Magazine Platform [D]. Beijing Institute of Graphic Communication, 2014. 孟祥兆.手机杂志平台内容 整合机制及优化策略研究[D].北京印刷学院.2014.

[4] Value-Added Services [DB/OL].(2015-06-18)[2015-07-07]. http://baike.baidu.com/link?url=9Hnz1jVOjRWPjT4zZN78xWoxE6P8X4lf2FIY5TAYJQO5jqzSjgiRBt2S9 Li9Bt_mpfxij5qyRSc8x_sidhhK. 增值服务

[5] Zhang Xianghong. On Management of A Periodical's Readership Relationship [J], Journal of Ningbo Polytechnic, 2011(4). 张向红.期刊 读者关系管理浅析 [J]. 宁波职业技术学院学报,2011(4).

[6] Ye Ping. On Content Integration and Form Innovation of Journals in Educational Industry [J]. Chinese Editors, 2008(2):40-43, 54. 叶萍.浅 议教育行业期刊的内容整合与形式创新 [J]. 中国编辑,2008(2):4043、 54 . 